\title{
Papel que desempeñan los servicios especializados en la respuesta a los problemas relacionados con el alcohol*
}

\author{
Bruce Ritson \\ Correspondencia: \\ Bruce Ritson. Department of Psychiatry. Edinburgh University. Royal Edinburgh Hospital. Scotland. bruceritson@netscapeonline.co.uk
}

\section{RESUMEN}

Durante los últimos cincuenta años los servicios especializados en problemas relacionados con el alcohol han experimentado una profunda transformación. En este texto se reseña brevemente cuál ha sido la evolución de los centros de tratamiento en el Reino Unido. Actualmente, la eficacia de los tratamientos a pacientes externos y la atención domiciliaria goza de excelente reputación. Este modelo de servicios implica una serie de derivaciones que incluyen la necesidad de formar y apoyar a los trabajadores de los servicios de atención primaria asegurándose, al mismo tiempo, de que no se pierda la red de tratamientos especializados, que son los que se dirigen a individuos con necesidades complejas. Finalmente, los cambios tienen que llevarse a cabo asumiendo que la respuesta social a los problemas relacionados con el alcohol debe ser mucho más amplia.

Palabras clave: alcoholismo, tratamiento, asistencia primaria, servicios especializados, Reino Unido.

\section{SUMMARY:}

Specialist Services for alcohol related problems have undergone enormous changes during the past 50 years. The evolution of treatment services in the UK is briefly outlined. The effectiveness of outpatient and domiciliary treatments is now well established. The implications for the pattern of services include the need to train and support primary care workers while ensuring that a network of specialist provision, particularly for individuals who have complex needs, is not lost. Finally, these changes need to occur against a background of a broader community response to alcohol related problems.

Keywords: aalcoholism, treatment, primary care, specialist treatment, United Kingdom

\section{INTRODUCCIÓN}

E sta ponencia analiza el papel que desempeñan los servicios especializados en el tratamiento de individuos con problemas relacionados con el alcohol y sus relaciones con los servicios de atención primaria. Aunque está centrada en el Reino Unido, refiere tendencias evidentes en muchos otros países.

\section{DESARROLLO DE LOS SERVICIOS ESPECIALIZADOS}

A principios de los años cincuenta los centros de tratamiento de Gran Bretaña mostraban poco interés hacia el alcoholismo o los problemas relacionados con el alcohol. El análisis de la situación de Gran Bretaña en aquella época muestra que el nivel de consumo de alcohol per capita era relativamente bajo en comparación con el que existe en la actualidad. El informe elaborado por el WHO Expert Committee (W.H.O. 1951) recomendaba la creación de programas para tratamiento del "alcoholismo", y Gran Bretaña respondió estableciendo unidades regionales para el tratamiento especializado del alcoholismo en hospitales.

¿En qué consistían estas primeras unidades especializadas? Casi todas estaban vinculadas a hospitales psiquiátricos. Generalmente se ingresaba a los pacientes para someterlos a tratamientos de unas seis semanas o más de duración, continuando después con un seguimiento posterior ya como pacientes externos. A lo que más importancia se concedía era a

\footnotetext{
* Basado en una ponencia presentada en el $28^{\circ}$ Congreso de Socidrogalcohol celebrado en Santander en abril de 2001
} 
la terapia de grupo, ya que imperaba la convicción de que el abuso del alcohol se manifestaba como resultado de problemas psicológicos del individuo que podían resolverse mediante psicoterapia apoyada, en algunos casos, con Antabuse. La importancia del trabajo en grupo también procedía de la filosofía de los Alcohólicos Anónimos, que en aquella época se habían consolidado como un grupo de auto ayuda clave para el apoyo y la recuperación de los alcohólicos. Todas estas unidades especializadas estaban dirigidas por psiquiatras. Los equipos incluían enfermeras y enfermeros, especialistas en psicología clínica y trabajadores sociales, y generalmente mantenían vínculos con AA y, en algunas ocasiones, con otras organizaciones de voluntarios como Councils on Alcohol, que empezaron a formarse en los años sesenta (Kessel and Walton 1965).

Diez años después, el carácter y las prioridades de estos servicios empezaron a cambiar rápidamente. El cambio estuvo propiciado en gran medida por los estudios y las valoraciones llevadas a cabo tanto en Londres -como las de Edwards y sus colegas(Edwards and Guthrie 1966) como en Edimburgo y otros lugares (Chick et al 1985). En ellos se demostró que la mayor parte de los tratamientos que recibían los pacientes internos que permanecían ingresados durante estancias prolongadas no eran significativamente más eficaces que los que se aplicaban a los pacientes externos. Más tarde se comprobó que los tratamientos breves a pacientes externos eran a menudo igual de eficaces que las terapias más complicadas. Todo esto coincidió con el desarrollo de una visión mucho más amplia del espectro de los problemas relacionados con el alcohol. El problema dejó de contemplarse desde la estrechez del concepto de alcoholismo y pasó a abarcar los perjuicios físicos y psicológicos derivados del consumo de alcohol que implica riesgos para la salud, una situación que contribuyó a que creciese significativamente el número de servicios especializados en tratamientos para pacientes externos al alcance de la mayoría de la sociedad. También se pudo observar que prácticamente sólo una de cada diez personas con problemas de bebida acudían a tratarse en servicios especializados, y que por lo tanto era necesario un espectro mucho más amplio de servicios para dar respuesta a toda la diversidad de problemas relacionados con el alcohol. Con ello quedó claro que había muchos individuos con problemas con la bebida que o bien intentaban salir adelante por sí mismos, generalmente como respuesta a las presiones sociales, o bien lo hacían con el apoyo en sus familias. Diversos informes llevados a cabo en distintas poblaciones demostraron que los individuos entraban y salían de las situaciones de consumo de alcohol nocivo o con riesgo para la salud, y que por eso era muy importante encontrar mecanismos socia- les que propiciasen cambios positivos (Roizen et al 1978).

\section{ATENCIÓN PRIMARIA}

También quedó claro que en los momentos de crisis, los bebedores con problemas contactaban con centros de atención primaria de muy diversos tipos, incluyendo Casas de Socorro y servicios de urgencias, hospitales generales de pacientes internos y psiquiátricos. La gente con problemas de bebida también acudía a centros de apoyo, servicios sociales y a tribunales y cárceles. Poco a poco fue evidenciándose que para proceder a la identificación y la aplicación de las primeras medidas de socorro a individuos con actitudes peligrosas o nocivas respecto a la bebida y para atender a aquellos que ya manifestaban una dependencia total, era necesaria la colaboración de toda una red de organismos.

El Maudsley Alcohol Pilot Project (MAPP) supuso una importante base conceptual para reflexionar sobre el primer nivel de respuesta a los problemas derivados del alcohol. Este proyecto estudió los factores que obstaculizaban la participación de los centros de atención primaria, así como de las posibles vías para solucionarlos. Plantearon que las dificultades que experimentaban los empleados de este tipo de servicios en este campo estaban relacionadas con tres factores subyacentes fundamentales. Eran los siguientes:

1. Inquietud acerca de su adecuación a la tarea por carecer de la información y las aptitudes necesa rias para identificar y responder a los bebedores.

2. Inquietud acerca de la legitimidad de la tarea, o sensación de inseguridad sobre si los problemas relacionados con la bebida caen o no bajo su responsabilidad profesional.

3. Inquietud acerca del apoyo en la tarea. Sensación de no tener un lugar al que acudir para recibir ayuda en los casos en los que no se sabe cómo responder a un problema relacionado con el alcohol.

Cuando los trabajadores de los servicios de atención primaria manifestaban estas sensaciones, el trabajo con bebedores conflictivos se desarrollaba en un clima de indecisión e inseguridad, una situación que acarreaba un escaso compromiso terapéutico. Los que se sentían más seguros trabajando con bebedores con problemas mostraban actitudes que elevaban el nivel de compromiso. Se trataba de personas que contaban con experiencia en el trabajo con problemas 
relacionados con el consumo abusivo de alcohol o que ya habían trabajado anteriormente en situaciones en las que habían contado con buenos equipos de apoyo. Los trabajadores de los servicios de atención primaria más seguros de sí mismos habían recibido una preparación en tratamientos y terapias de apoyo y poseían conocimientos clínicos sobre el alcohol y los problemas relacionados con el mismo.

A raíz del conocimiento de estos datos los servicios especializados empezaron a dedicar más tiempo a acercarse a la sociedad, desarrollando la formación y apoyando a la red de centros de atención primaria. Se crearon grupos locales de tratamiento integral del alcoholismo en muchas zonas, que actuaban a modo de consultorios, integrados por enfermeras y enfermeros, trabajadores sociales, especialistas en terapias de apoyo y, a menudo, psicólogos y psiquiatras. Al irse extendiendo por todo el país, este tipo de equipos locales para el tratamiento de los problemas relacionados con el alcohol obtuvieron un éxito desigual (Stockwell and Clement 1988). Tuvieron muchos problemas a la hora de influir en el comportamiento de sus colegas que se dedicaban a la atención primaria, y muchos de ellos acabaron derivando hacia la provisión de servicios directos que, aunque son de una gran utilidad, sólo consiguen llegar a una reducida proporción de clientes. (Para un análisis excelente y detallado de esta evolución en Inglaterra, véase Thom 1999).

El modelo de servicios que se adoptó en Gran Bretaña en esta época estuvo influido por un informe del Institute of Medicine (1990) de los Estados Unidos que concedía una gran importancia al hecho de que la educación sanitaria y el tratamiento en centros de atención primaria podía servir de ayuda a muchos de los individuos que tienen comportamientos nocivos respecto a la bebida o que consumen alcohol en cantidades que suponen un riesgo para la salud, y que sólo una minoría requería tratamientos especializados intensivos o rehabilitación a largo plazo. La eficacia y el reducido coste de los tratamientos breves de este tipo en los centros de atención primaria era clarísima (Effective Health Care 1993). En este sentido, el papel de los médicos de familia cobró una gran importancia en el desarrollo de las iniciativas que abogaban por orientar los servicios hacia el diagnóstico precoz y los tratamientos breves para la mayoría de las personas con problemas relacionados con el alcohol.

Aunque muchas personas reconocieron las ventajas de la atención primaria en este sentido, también resultó difícil convencer a numerosos médicos de familia, porque veían que la orientación de algunos servicios hacia los tratamientos domiciliarios supondría tener que cargar su tiempo con más tareas. En la Fig. 1 pueden verse algunas de las ventajas que proporciona la atención a nivel primario junto a una lista en la que aparecen las desventajas y resistencias que se perciben.
Fig 1.

\section{Ventajas e inconvenientes de las intervenciones eficaces contra el alcohol en Servicios de Atención Primaria}

\begin{tabular}{|c|c|}
\hline$\underline{\text { VENTAJAS }}$ & $\underline{\text { INCONVENIENTES }}$ \\
\hline Accesibilidad & Actitud \\
\hline Aceptabilidad & Conocimiento \\
\hline Credibilidad & Estereotipos \\
\hline No Estigmatización & Tiempo \\
\hline Contacto frecuente & $\begin{array}{c}\text { Personas que atienden (a } \\
\text { menudo pocos adultos de } \\
\text { sexo masculino) }\end{array}$ \\
\hline Reproducibilidad & $\begin{array}{r}\text { Recompensas } \\
\text { Prioridades }\end{array}$ \\
\hline
\end{tabular}

La atención primaria tiene la ventaja de ser mucho más accesible en la mayoría de las comunidades urbanas y rurales. Su visita no conlleva la carga de estigmatización que supone la asistencia a "Servicios contra el Alcoholismo" especializados, y por ello muchos la consideran más aceptable. La credibilidad de los centros de atención primaria está completamente afianzada en la comunidad, y no tienen que soportar el peso de estigmas de ningún tipo. Suelen ser el primer punto de contacto de los pacientes, sea cual sea el carácter de su enfermedad, y están bien situados en lugares accesibles y convenientemente protegidos. Por último, si el tratamiento se prueba en una zona y se demuestra su eficacia, puede reproducirse rápidamente en todo el espectro de centros de atención primaria del conjunto del país.

Sin embargo, también existen inconvenientes que dificultan el trabajo eficaz en este tipo de centros, incluyendo la actitud de los médicos de familia y de las enfermeras y enfermeros hacia los individuos que tienen problemas con el alcohol. La falta de conocimiento que muestran a la hora de responder a las necesidades de los alcohólicos, unida a la existencia continuada de "estereotipos" sobre estas personas puede impedir la percepción de los primeros síntomas. Por ello todavía existe una tendencia a identificar sólo casos extremos en los que la adicción está completamente asentada y el declive social ya ha comenzado. 
Los médicos de familia han manifestado su preocupación por el tiempo que supone este trabajo, la falta de prioridad que se le concede y, en algunos casos, cuando se toman medidas preventivas más que "tratamientos activos", la inexistencia absoluta de compensaciones económicas. En los pacientes que acuden a los Centros de Salud de muchas zonas se observa una escasa representación de jóvenes de sexo masculino, a pesar de que este grupo es especialmente propenso a conductas de consumo alcohólico de riesgo. Puede que estén más dispuestos a acudir a servicios de salud ocupacionales.

Recientes estudios llevados a cabo en el Reino Unido han mostrado que los médicos de familia que consideran positivo estar involucrados en tareas de este tipo siguen siendo minoría (Deehan at el 1998). A los servicios especializados todavía les queda un largo camino por recorrer, apoyando y fomentando servicios de atención primaria, antes de que este enfoque consiga ser aceptado mayoritariamente. En Gran Bretaña se están realizando una serie de proyectos de investigación sobre el tipo de marketing más adecuado para apoyar la postura que defiende los tratamientos breves, centrándose en averiguar buenos métodos para que los grupos de especialistas consigan que se elijan, y para incrementar su eficacia en pacientes/clientes individuales. Probablemente sería conveniente que las enfermeras y enfermeros de atención primaria asumiesen una gran parte de esta tarea.

\section{TRATAMIENTOS ESPECIALES}

Una de las tareas más importantes de los servicios especializados ha sido el desarrollo y la promoción de tratamientos eficaces. La valoración de la eficacia en el tratamiento de los problemas relacionados con el alcohol goza cada vez de un respaldo mayor. Antiguamente los métodos de mayor eficacia probada eran los que menos se adoptaban en centros de tratamiento. Esta situación cambió rápidamente. En este campo destaca la influencia del trabajo de Miller y sus colegas (1998), que revisaron los estudios sobre tratamientos y los evaluaron y juzgaron tanto en términos de calidad de la investigación como en su eficacia. Los enfoques que gozan de mayor apoyo científico son: entrevistas motivacionales, formación en la adquisición de aptitudes sociales, terapia para refuerzo social $y$, entre los tratamientos a base de fármacos, el uso de Acamprosate o antagonistas de opiáceos (principalmente Naltrexona) y Antabus (Disulfiram) siempre que exista una buena supervisión. Sin embargo, el panorama evoluciona constantemente, a medida que se van desarrollando nuevas investigaciones. Por el momento no se tiene en cuenta el tipo de tratamiento, la importancia de las características del terapeuta o el factor fundamental del deseo del paciente a participar en la terapia y su conformidad con ella. Estos factores son de crucial importancia, y tanto para los servicios especializados como para el resto. Los datos reunidos también subrayan la importancia de la formación y la supervisión para asegurar que se pueda identificar y resulte aceptable lo que se pretende que ocurra en la terapia.

Todos los tratamientos que hemos citado más arriba pueden aplicarse a pacientes externos y muchos pueden proporcionarse a través de servicios generales, pero también requieren cierto conocimiento especializado y apoyo si pretenden ser eficaces.

\section{DESINTOXICACIÓN DOMICILIARIA}

Otra consecuencia del desarrollo de grupos locales de tratamiento integral del alcoholismo ha sido la eficacia demostrada y la extensión del uso de la desintoxicación domiciliaria. Actualmente se trata de una práctica muy extendida en Gran Bretaña, con enfermeras y enfermeros especialistas en medicina comunitaria que ayudan a los pacientes a dejar el alcohol en casa, supervisando la medicación correcta y la terapia a base de vitaminas y poniéndoles en contacto con tratamientos externos y apoyos a más largo plazo. (Stockwell et al 1986). Actualmente sólo una minoría de pacientes que muestra síntomas de recaída necesita internamiento hospitalario, la mayoría de las veces en casos en los que existe riesgo de acceso de recaída, delirium tremens, salud extremadamente deteriorada o falta de respaldo social. La creciente disponibilidad de enfermeras y enfermeros especialistas en alcoholismo y psiquiatría comunitaria ha contribuido a transformar el modelo de los servicios especializados.

El desarrollo y la experimentación de nuevos tratamientos psicológicos, sociales y farmacológicos seguirá siendo una de las tareas principales de los servicios especializados. Después se aseguran de que estas técnicas pasen a sus colegas de los centros de atención primaria. Pero las técnicas no se regalan sin lo que podría llamarse servicio "post venta" -manteniendo, apoyando y ayudando a los servicios de atención primaria sobre todo en los temas más complejos que, por ejemplo, surgen en clientes/pacientes con necesidades complejas o diagnóstico doble.

En muchos servicios se observa una tendencia al conservadurismo -conservar lo que es familiar y sentirse incómodo con el cambio es un fenómeno normal y comprensible. Los servicios especializados en alcoholismo no son una excepción y muchos de los cambios experimentados en los últimos años que hoy día se extienden por toda Europa no han sido adoptados sin dificultades y descontento. Al personal especializa- 
do, que ha recibido una formación en técnicas muy específicas, le resulta muy duro deshacerse de ellas y aprender otras nuevas, y frecuentemente se necesita ayuda y apoyo para llevar a cabo la transformación. El personal clínico que desea trabajar con clientes con problemas con el alcohol es una mercancía preciosa, y a la hora de abandonar antiguas técnicas y desarrollar nuevas aproximaciones merece el máximo estímulo y sustento.

\section{RESPUESTA SOCIAL}

No sirve de mucho proporcionar servicios para el rescate para aquellos que padecen hábitos nocivos respecto a la bebida si la sociedad no se enfrenta paralelamente a toda la gama de fuerzas que se encuentran en el origen de los problemas. En esta lógica se han desarrollado una serie de proyectos centrados en la respuesta de la sociedad a los problemas relacionados con el alcohol, muchos de ellos promovidos por W.H.O. (Ritson 1996).

Son muchos los factores de la vida cotidiana en sociedad que pueden aumentar o disminuir los peligros de la bebida, como la disponibilidad, la relación entre el precio y los ingresos disponibles: el papel del alcohol en las políticas de empleo, legislación de tráfico en relación con la bebida, y funcionamiento, formación del servicio y tipo de gestión en bares, pubs, restaurantes y otros lugares en los que existe bebida, temas de seguridad pública y muchos otros. Los municipios tienen una gran responsabilidad a la hora de asegurar el desarrollo de políticas públicas sanas, y ello implica planes sensatos de actuación sobre el consumo de alcohol. Los servicios especializados desempeñan un papel importante, garantizando la idoneidad de los planes de actuación en curso. También pueden encontrarse con que hay áreas determinadas en las que tienen que implicarse activamente para conseguir que se lleve a cabo la planificación y que la sociedad tome conciencia de que inevitablemente el alcohol es un factor fundamental a la hora de conformar la calidad de vida de la comunidad. (Para más detalles, vid. Hannibal et al 1995)

\section{CONCLUSIÓN}

Los servicios especializados se encuentran en una etapa muy importante de su desarrollo. Tienen la responsabilidad de "descubrir" y fomentar técnicas de eficacia probada a sus colegas de los servicios de atención primaria para que los puedan emplear y llegar al mayor número posible de personas de forma accesible, evitando los prejuicios e intentando ser competentes desde el punto de vista de los costes. También tienen que asegurarse de que los centros de atención primaria están bien preparados y cuentan con los apoyos necesarios sin que se les haya privado de su poder. Con este tipo de aventuras conjuntas se aprende tanto de la experiencia de la atención primaria como de los equipos de especialistas. Existen importantes tareas a desarrollar: apoyo continuo: tratamiento de pacientes con necesidades complejas, como los casos de doble diagnóstico (por ejemplo, los que conllevan riesgos de auto lesiones): organización de servicios eficaces de desintoxicación, comunitarios y en hospitales, para personas con dificultades especiales. Los servicios especializados también forman parte de la red de apoyo de las personas con problemas relacionados con el alcohol, casi nunca son los únicos servicios especializados en este campo. Finalmente, su papel es muy importante a la hora de defender la seguridad de que los que tengan problemas con el alcohol no sean rechazados o estigmatizados una vez más, y pueden influir en los Alcohol Action team para contribuir a la comprensión ponderada de los daños y los beneficios que supone el alcohol para la comunidad.

\section{BIBLIOGRAFÍA}

1. Chick J., Ritson B., Connaughton J. (1988). Advice versus extended treatment for alcoholism: a controlled study. British Journal of Addiction 83, 159-70.

2. Deehan A., Templeton I., Taylor C., Drummond C., and Strang J (1998). How do GPs manage alcohol misusing patients. Drug and alcohol review. 17: 259-66.

3. Edwards G and Guthrie S (1966). A comparison of inpatient treatment and out-patient treatment of alcohol dependence. Lancet 1, 467-8.

4. Effective Health Care Research Team (1993). Brief interventions and alcohol use. Effective Health Care Bulletin 7: 1-13.

5. Hannibal J., Van Iwaarden M., Gefou Madianou D., Moskalewicz J., Ritson B. and Rud M (1995). Alcohol and the Community WHO (Euro) Copenhague.

6. Institute of Medicine (1990). Broadening the base of treatment for alcohol problems. National Academy Press. Washington.

7. Kessel N., Walton H.J. (1965). Alcoholism. Hammondsworth: Penguin.

8. Ritson B (1996). Community and municipal action on alcohol. WHO (Euro) Copenhague.

9. Roizen R., Cahalan D., Shanks I (1978). Spontaneous remission among untreated problem drinkers. En Longitudinal Research on Drug Use (ed. D Kandel). Wiley. Nueva York. 
10. Stockwell T and Clement S. (1988). Community alcohol teams: a review of studies evaluating their effectiveness with special reference to the experience of other community teams. DHSS, Londres.

11. Stockwell T, Bolt E., Hooper J., (1986). Detoxification from alcohol at home managed by general practitioners. British Medical Journal 292, 733-735.
12. Thom B (1999). Dealing with drink. Free Association Books. Chaddington.

13. World Health Organisation (1951). Report on the first session of the alcoholism sub-committee. Expert Committee on mental health. Tech. Rep. Series 42: WHO Ginebra 